\title{
Couloir prothétique et gérodontologie : traitement simple pour une stabilité optimum
}

\section{RÉSUMÉ}

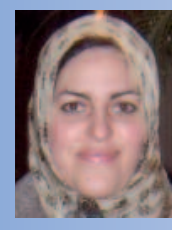

Anissa REGRAGUI

Spécialiste en prothèse amovible. Université Mohamed V

Faculté de médecine dentaire de Rabat, Avenue Allal El Fassi,

Rue Mohammed Jazouli,

Madinat Al Irfane,

BP 6212,

Rabat - Instituts, Maroc.

Amal SEFRIOUI

Professeur assistante en prothèse amovible, Université Mohamed V

Faculté de médecine dentaire de Rabat.

Faïza BENFDIL

Professeur agrégé en prothèse amovible, Université Mohamed $\mathrm{V}$,

Faculté de médecine dentaire de Rabat.

Amar BENAMAR

Professeur d'enseignement supérieur, en prothèse amovible,

Université Mohamed V,

Faculté de médecine dentaire de Rabat.

\section{Ahmed ABDEDINE}

Professeur d'enseignement supérieur, Chef du service de prothèse amovible, Centre de consultation et des traitements dentaires de Rabat-CCTD Université Mohamed V,

Faculté de médecine dentaire de Rabat.
Le processus du vieillissement s'accompagne le plus souvent, au niveau buccal, d'un rétrécissement du couloir prothétique en rapport avec l'importance de la résorption osseuse et l'envahissement des éléments périphériques ; la situation devient, de ce fait, défavorable pour la réalisation prothétique.

Le recours à la piézographie en prothèse complète permet de mieux respecter la physiologie buccale du sujet âgé tout en assurant un maximum de stabilité prothétique.

La piézographie relève en effet quatre défis : restituer au visage son aspect naturel et harmonieux, proposer un plan d'occlusion, rechercher une dimension verticale d'occlusion, conforter une relation intermaxillaire apte à assurer la fonction masticatoire.

Un protocole rationnel, ergonomique et individualisé s'avère particulièrement adapté aux cas difficiles, permettant ainsi de bien définir le volume prothétique dévolu à l'emplacement des dents artificielles. C'est ce que nous allons essayer d'illustrer, à travers deux cas cliniques. 


\section{Introduction}

$>$

Même si la réhabilitation prothétique complète est un acte fréquent, cette thérapeutique reste un exercice prothétique et psychologique complexe.

L'allongement de la durée de vie et le vieillissement de la population qui en résulte font que les situations de crêtes mandibulaires plates, voire négatives, sont de plus en plus nombreuses. Lorsque le recours à l'implantologie n'est pas possible, la réalisation de prothèses amovibles complètes bi-maxillaires s'avère très difficile, tant pour le clinicien que pour le prothésiste. La piézographie mandibulaire est alors une technique de choix qui va faciliter le travail du praticien et permettre au patient d'accepter plus facilement ses prothèses par l'amélioration de sa qualité de vie [1].

En effet, dans les situations de crêtes plates, le respect de la triade de Housset (rétention, sustentation, stabilisation) s'avère très complexe

\section{Couloir prothétique}

\section{Définition}

Le couloir prothétique ou l'espace prothétique disponible n'est ni une zone neutre ni un espace passif, c'est l'espace édenté où la résultante des forces horizontales développées par la langue et la sangle buccinato-labiale ne doit pas dépasser la rétention globale des prothèses (Klein, 1988) [1].

Selon R. Devin, c'est le volume dans lequel on doit inscrire la prothèse pour lui assurer une stabilité maximale. Il est matérialisé par l'enregistrement dans une pâte plastique - piézo- à réaliser, car la surface développée des crêtes résiduelles est très faible. Les repères habituels de montage des dents prothétiques n'existent plus, le modelage obtenu par la piézographie trouve alors toute son utilité dans la matérialisation de l'espace dévolu à ce montage $[2,3]$. La physiologie musculaire qui a permis I'obtention de ce volume n'est plus alors considérée comme une gêne ou un obstacle mais comme une aide qui permet l'optimisation de l'équilibre prothétique et guide le prothésiste dans le positionnement des dents prothétiques.

Cette technique va permettre de situer le couloir prothétique de façon optimale pour que la musculature périphérique favorise la stabilisation et la rétention afin de compenser le déficit de surface des crêtes.

La forme donnée par la physiologie va permettre de restaurer l'équilibre fonctionnel dans un cadre esthétique optimal $[4,5]$.

graphe des pressions exercées par les différents groupes musculaires antagonistes au niveau des arcades dentaires [6].

\section{Anatomie [7, 8]}

Le couloir prothétique est compris entre les quatre murs suivants (fig. 1 a et $b$ ) :

- mur inférieur : il correspond à la surface d'appui statique des crêtes ;

- mur externe (ou mur vestibulaire) : il se compose de six muscles : le masséter dont les insertions basses limitent le couloir pro- 

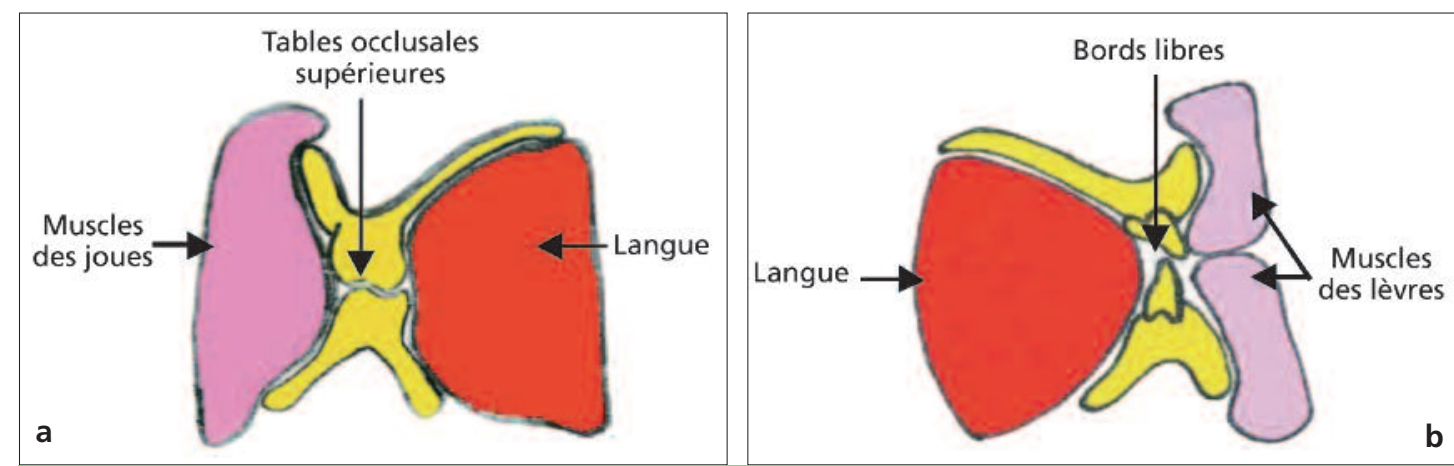

Fig. 1 a et b coupe frontale (a) et sagittale (b) montrant les structures délimitant le couloir prothétique.

thétique du côté distovestibulaire ; le buccinateur qui s'insère en arrière sur le ligament ptérygo-maxillaire, en haut sur le maxillaire supérieur et en bas sur la mandibule en regard des trois dernières molaires; l'orbiculaire des lèvres constitué de fibres concentriques réparties en deux groupes (I'orbiculaire externe et l'orbiculaire interne) ; le carré du menton qui s'insère sur la mandibule entre le rempart alvéolaire de l'incisive latérale et l'éminence canine; la houppe du menton insérée sur la partie basse de la bosse canine; et le modiolus "véritable nœud musculaire " constitué de six muscles : le buccinateur, I'orbiculaire des lèvres, le risorius, le grand zygomatique, le releveur de la lèvre supérieure et l'abaisseur de la lèvre inférieure. II se situe à côté et légèrement au-dessus de la commissure ;
- mur interne : il est créé par trois muscles : le ptérygoïdien médial dont les insertions basses se situent sur la face interne de la branche mandibulaire et son trajet est symétrique à celui du masséter ; le mylohyoïdien qui s'insère latéralement sur la ligne oblique interne et au centre avec les fibres de son homologue pour former le plancher de la bouche; la langue constituée de dix-sept muscles répartis en deux groupes : groupe intrinsèque et groupe extrinsèque ; dans ce dernier, trois muscles ont une incidence particulière sur la prothèse : le palatoglosse et le styloglosse postérieurement et le génioglosse antérieurement ;

- mur supérieur : il est constitué par les tables occlusales des dents résiduelles. Selon Merkeley (1966), le plan d'occlusion se situe en regard du sillon du buccinateur et de la limite entre la partie papillée et dépapillée de la langue.

\section{Influence de la sénescence sur le couloir prothétique}

L'espace occupé par les dents et les procès alvéolaires non résorbés se transforme avec la perte des dents et les phénomènes inhérents à la sénescence, en un espace plus réduit appelé espace prothétique. La sénescence provoque une diminution importante de la dimension 
verticale ; ceci entraîne un plissement des joues qui se traduit par l'apparition de bajoues et l'invagination de la sangle buccinato-linguale. L'impact esthétique est très important.

Parallèlement, la langue comble l'espace libre en s'étalant : elle n'est plus contenue par les remparts dentaires et s'étale donc sur l'ensemble de la largeur de la cavité buccale, d'une paroi jugale à l'autre transversalement et jusqu'à la lèvre en avant [9].

Chez le sujet édenté, le trou mentonnier se localise parfois au sommet de la crête osseuse et la ligne oblique interne également; elle se termine par une saillie osseuse à sa partie distale, la tubérosité linguale. Les apophyses géni se situent sur cette crête résiduelle.
La sénescence altère toutes les structures anatomiques et physiologiques et, en particulier, les surfaces d'appui. La résorption de l'os crestal et la migration relative des insertions des muscles mylohyoïdiens et buccinateurs vers le sommet de la crête résiduelle sont sources de difficultés dans la réalisation de prothèse adjointe, surtout à la mandibule (fig. 2).

La muqueuse s'amincit et les muscles perdent leur tonicité. De plus, en vieillissant, I'ensemble du système neurovégétatif s'altère [10].

Devant ces conditions, il apparaît nécessaire de tenter une exploitation maximale des surfaces d'appui et des organes paraprothétiques via une empreinte piézographique réalisée suite à

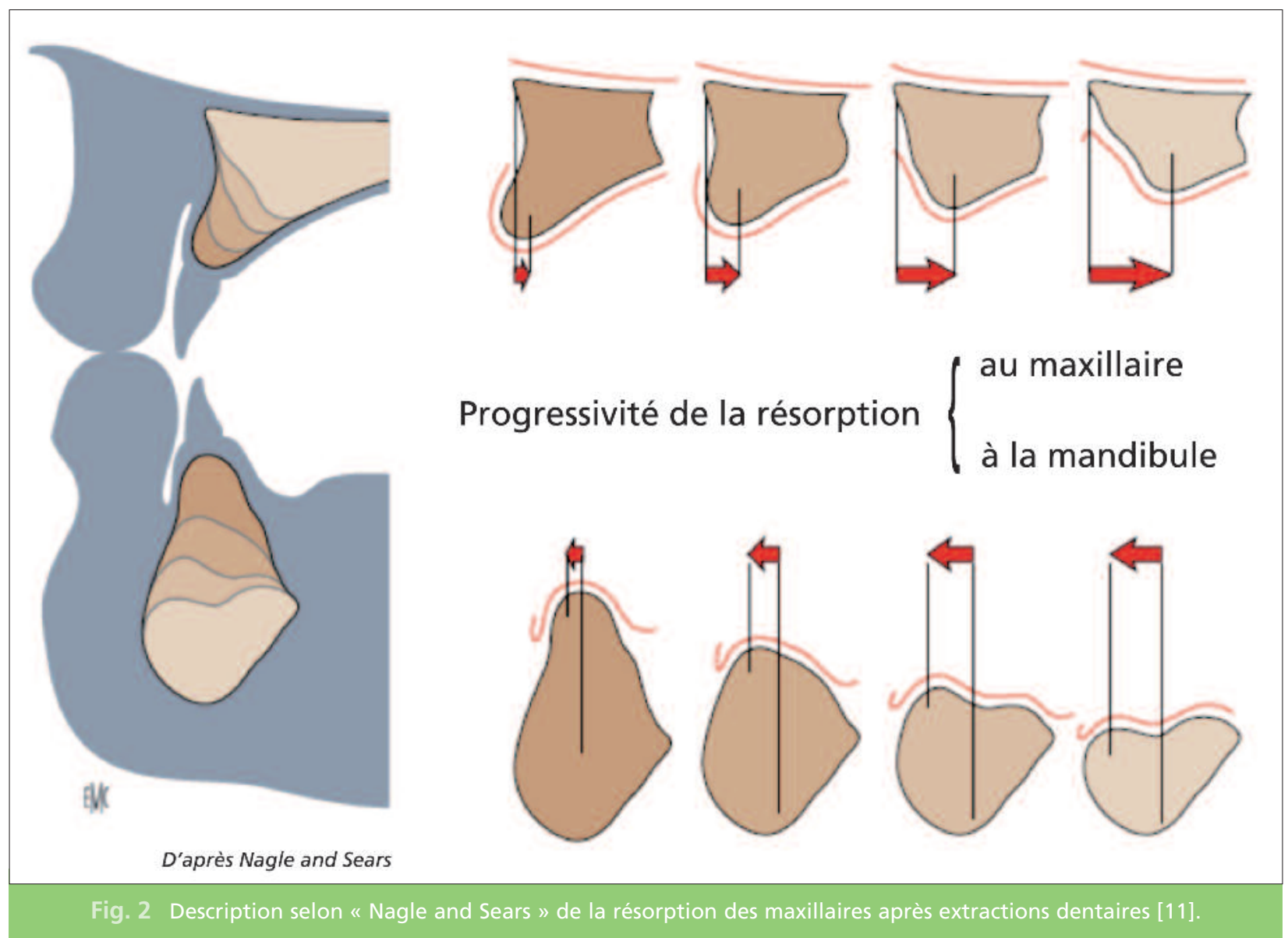


I'empreinte secondaire à la mandibule, et avant le modelage de la cire d'occlusion; elle s'intègre donc au sein d'un ensemble théra- peutique afin de répondre aux exigences physiologiques, voire pathologiques rencontrées dans la bouche de notre patient [11].

\section{Apport de la piézographie dans le modelage du couloir prothétique}

Issue de l'adjonction de deux verbes grecs piézein " presser » et graphein « inscrire », la piézographie est une technique d'inscription des pressions produites par la langue d'une part, et par la sangle buccinato-labiale, d'autre part, dans un matériau plastique disposé en bourrelet, comme une arcade dentaire, sur la plaque base mandibulaire.

P. Klein a apporté ce terme au domaine odontologique pour désigner le modelage par pression d'un matériau plastique. Ce modelage résulte des pressions qui déterminent l'espace prothétique disponible au cours de la fonction et au repos; il permet au patient d'exprimer des paramètres musculaires, articulaires, fonctionnels et esthétiques spécifiques. Ainsi, une prothèse issue d'une maquette piézographique permet au patient une plus grande liberté dans ses possibilités fonctionnelles quotidiennes $[12,13]$. Les phénomènes physiques d'adhésion, cohésion et pression atmosphérique ne doivent pas être limités à l'interface intrados prothétique et la surface d'appui basale, mais doivent être augmentés par le contact généralisé des muqueuses revêtant les muscles avec l'extrados prothétique.

Le contact permanent des muqueuses de la langue, des joues et des lèvres avec les sur- faces polies prothétiques réduit la formation de dépôts tartriques à ce niveau et diminue donc les risques de prolifération du Candida albicans dans la cavité buccale [14].

Lors de la mastication, les particules alimentaires ont moins tendance à s'insinuer entre l'extrados prothétique d'une part et la langue et les joues d'autre part; ainsi, lors de cette fonction, la stabilité prothétique risque moins d'être perturbée.

L'empreinte piézographique est une empreinte dynamique et fonctionnelle. Les fonctions sollicitées pour ce moulage du couloir prothétique sont le plus souvent la phonation (choisie par P. Klein du fait que la phonation est la fonction buccale la moins affectée par la perte des organes dentaires et paradentaires et la fonction orale la plus développée) et la déglutition (M.R. Heath conclut que les forces linguales ont été plus grandes que les forces vestibulaires).

Les forces modelantes imposées par la langue et les sangles buccinato-labiales modèlent la résine plastique dont les excès fusent vers les zones de moindre pression.

On obtient ainsi un volume de résine dans la zone d'équilibre musculaire, entre la langue et la sangle buccinato-labiale $[15,16]$. 


\section{Illustrations cliniques}

\section{Cas 1 : enregistrement}

$$
\begin{aligned}
& \text { piézographique } \\
& \text { simultané aux thiocols }
\end{aligned}
$$

Il s'agit d'un patient de 66 ans, qui se présente au service de prothèse adjointe pour une réhabilitation prothétique.

L'examen exobuccal montre un affaissement de l'étage inférieur avec un prognathisme mandibulaire.

L'examen endobuccal révèle des reliefs anatomiques très résorbés au niveau mandibulaire altérant ainsi la stabilité et la rétention prothétiques (fig. 3).

L'examen des prothèses met en évidence une conception prothétique erronée puisque la dimension verticale d'occlusion est diminuée, la relation centrée n'est pas respectée ; on assiste plutôt à un articulé inversé (fig. 4).

L'intrados des prothèses est le siège de gros amas de plaque dentaire et de tartre. Les dents prothétiques sont très abrasées.

Devant le grand nombre d'échecs rencontrés dans la réhabilitation prothétique des crêtes

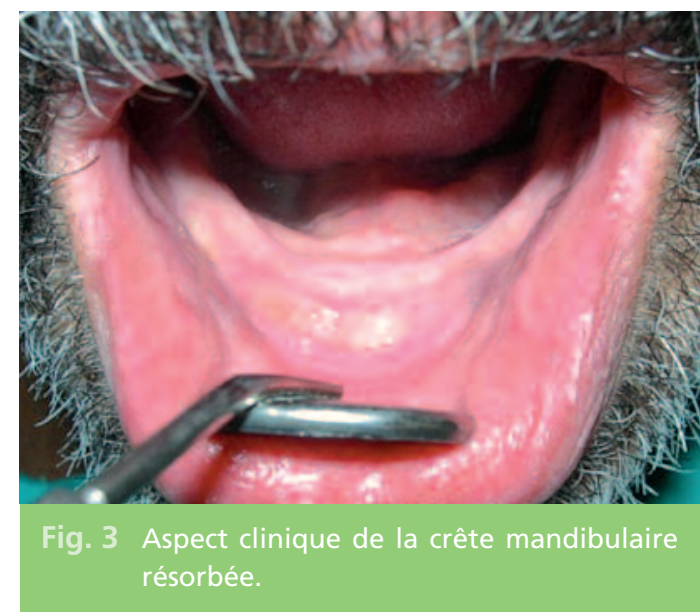

plates ou négatives, les techniques classiques s'avèrent parfois insuffisantes, en prothèse amovible complète mandibulaire, d'où l'intérêt de la piézographie grâce à des enregistrements de l'espace prothétique guidant ainsi toute la construction prothétique, à savoir : la détermination du volume de la prothèse dans les trois plans de l'espace, la détermination de la dimension verticale d'occlusion, la détermination d'une relation maxillo-mandibulaire horizontale et le montage des dents à l'intérieur du volume enregistré [13].

\section{> La base piézographique}

Comme la piézographie se fait en plusieurs temps, il est nécessaire de réunir les enregistrements par une base qui doit être réduite au strict minimum afin de ne pas interférer avec le jeu des organes paraprothétiques.

Cette base peut être un fil préformé selon la configuration de l'arcade, épousant la crête et s'arrêtant à $10 \mathrm{~mm}$ des trigones rétromolaires. Quand une base en résine est utilisée, elle doit satisfaire en bouche les tests de Herbst, les tests phonétiques de Devin et le patient doit

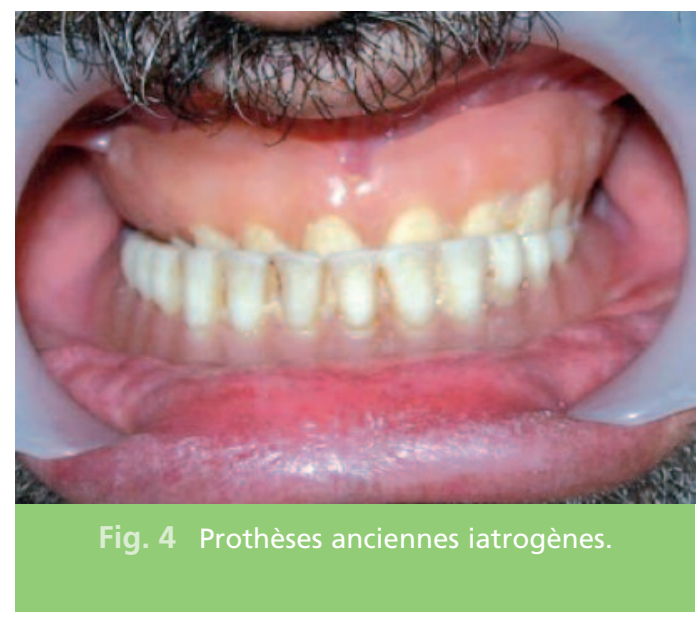



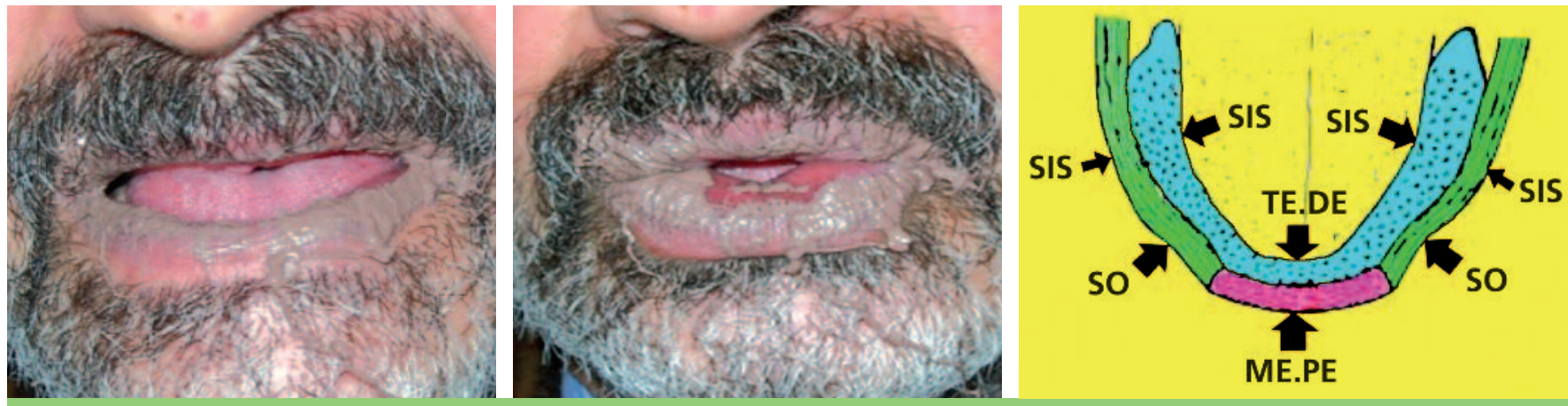

Fig. 5 a à c Prononciation des phonèmes -SIS- -SO- -TE- -DE- -ME- -PE-

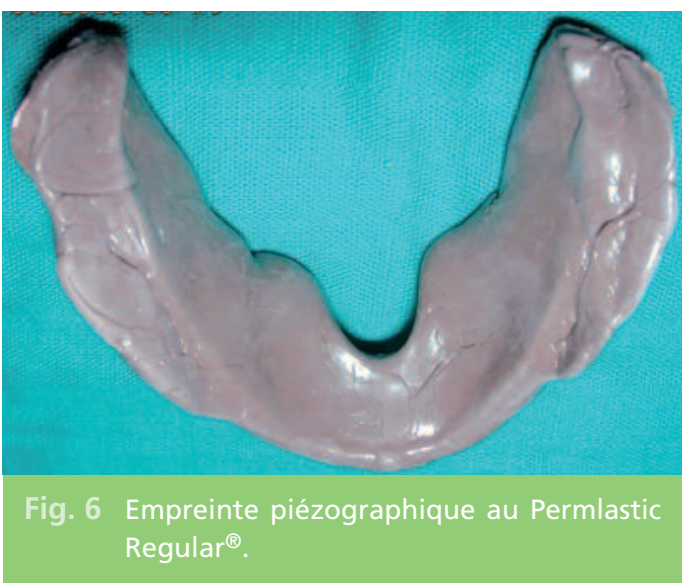

pouvoir parler avec aisance sans que la base ne quitte son siège [14].

\section{$>$ Le matériau}

Le matériau idéal doit répondre aux critères prérequis de stabilité et de fidélité. II doit, en outre, présenter un temps de plasticité suffisamment lent pour permettre les diverses manipulations. Nous avons utilisé un thiocol dense type Permlastic Regular ${ }^{\circledR}$.

Des empreintes secondaires maxillaire et mandibulaire sont réalisées ainsi que la maquette d'occlusion supérieure et le porte-empreinte individuel inférieur guidant la piézographie.

Au niveau mandibulaire, un polysulfure de moyenne viscosité de type Permlastic Regular ${ }^{\circledR}$ est utilisé.
Il est possible de ne badigeonner qu'une seule face à la fois, mais nous avons préféré stimuler de façon symétrique la proprioception linguale et l'extéroception jugale et labiale en appliquant le produit simultanément sur les faces vestibulaires et linguales de l'extrados.

La phonation est choisie comme vecteur du modelage piézographique mandibulaire en faisant prononcer au patient certains phonèmes par la répétition d'une séquence type (sis, sis, so, te, pe, de) jusqu'à ce que la pâte (Permlastic Regular ${ }^{\circledR}$ ) ne soit plus modifiable (fig. 5 a à c et fig. 6).

La langue exerce des forces centrifuges qui agissent sur les bords latéraux et la partie antérieure de l'empreinte.

Les forces centripètes sont effectuées en majorité par le buccinateur et par les lèvres en agissant sur la partie vestibulaire de la piézographie. Le patient est ensuite invité à parler, à déglutir et à effectuer les différents mouvements fonctionnels pour éliminer les excès du matériau et libérer le jeu des différents muscles concernés.

Mais il est impératif que la prise finale se déroule alors que la cavité buccale est au repos, pour appréhender la position d'équilibre musculaire $[14,15,17]$.

Deux clés en silicone sont réalisées pour déterminer le couloir prothétique où la résultante 
des forces horizontales développées par la langue et la sangle buccinato-labiale ne doit pas dépasser la rétention globale des prothèses (fig. 7).

Le rapport intermaxillaire est enregistré ; la maquette d'occlusion mandibulaire utilisée doit s'inscrire dans le couloir prothétique en respectant les clés en silicone ainsi que le montage des dents prothétiques (fig. 8).

Une fois polymérisées, les nouvelles prothèses sont essayées en bouche en assurant une stabilité prothétique admirée par le patient (fig. 9).

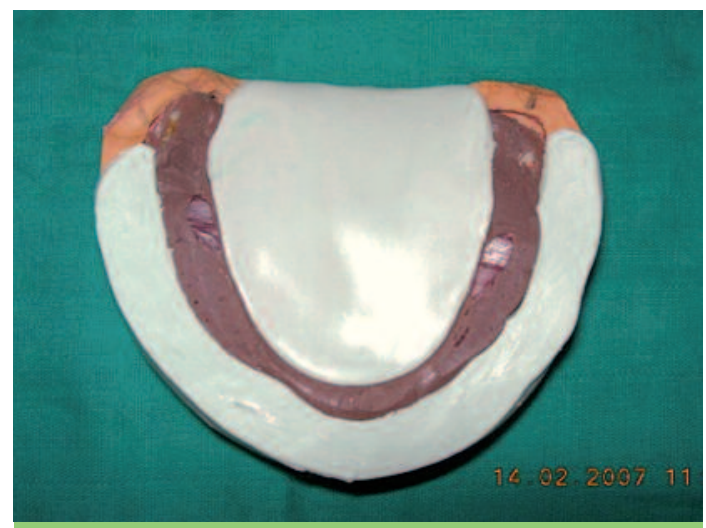

Fig. 7 Réalisation de deux clés en Optosil ${ }^{\circledR}$ en vestibulaire et en lingual.
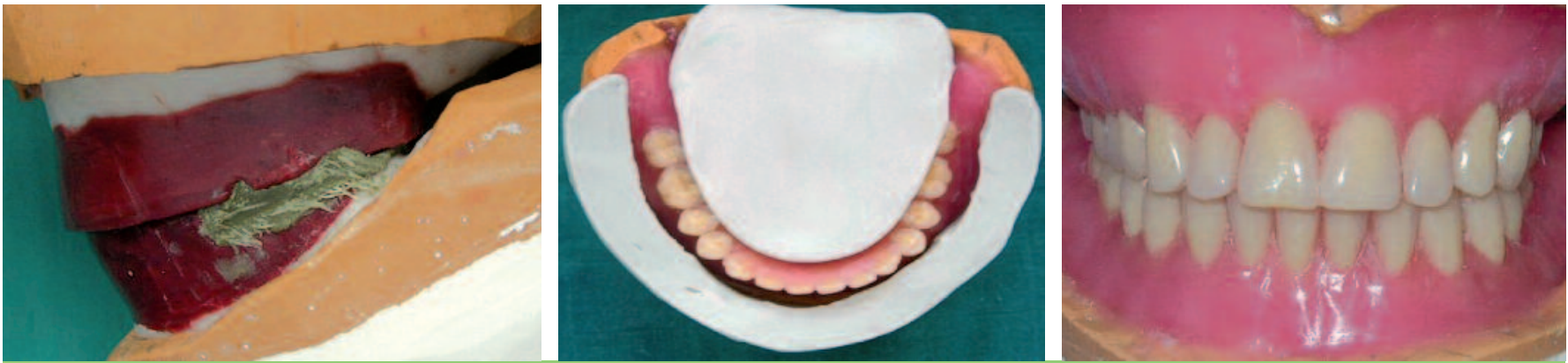

Fig. 8 a à c Montage piézographique.

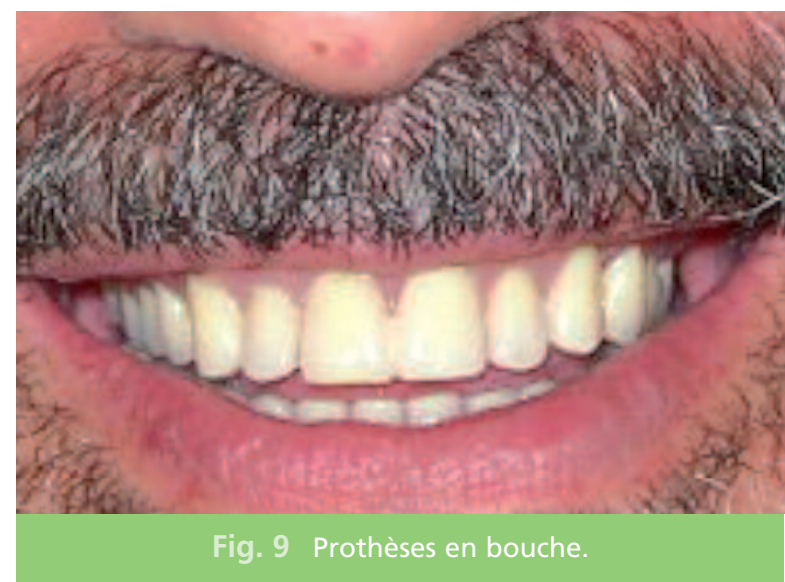




\section{Cas 2 : enregistrement piézographique secteur par secteur aux résines retard}

Dans le souhait de restaurer sa fonction masticatoire et de remplacer ses prothèses défectueuses, le patient se présente dans le service.

Les prothèses actuelles du patient présentent une abrasion des dents prothétiques entraînant un effondrement de la dimension verticale d'occlusion (DVO) et une perte de la relation centrée (RC) (fig. 10).

Devant la résorption mandibulaire importante, la réhabilitation prothétique du patient fait appel à la piézographie (fig. 11).

Des empreintes primaires sont réalisées et un porte empreinte individuel mandibulaire est
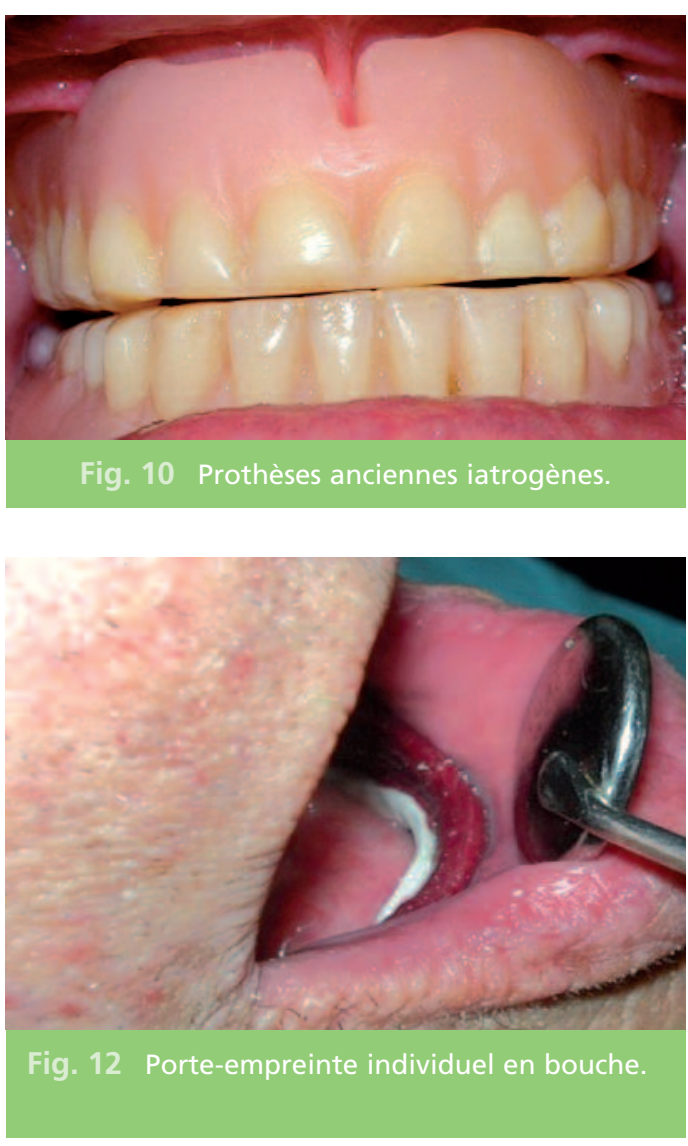

réalisé en résine avec un bourrelet très fin (lame de Brill). En aucun cas le bourrelet ne devra être en contact avec la langue ou avec la sangle buccinato-labiale (fig. 12).

Dans un premier temps, les surfaces d'appui primaires sont enregistrées par de la résine à prise retardée (pré-empreinte) (fig. 13).

Puis, un premier modelage du buccinateur et de la langue est effectué ; le patient est alors prié de répéter après l'opérateur cinq fois "SIS » et une fois «SO ». Ces phonèmes sont renouvelés jusqu'à ce que le matériau devienne suffisamment rigide pour ne plus être déformé par les organes buccaux.

La durée optimum du modelage se situe entre trois et six minutes, durée maximum pendant laquelle le patient peut rester sans déglutir (il faut absolument éviter toute déglutition afin
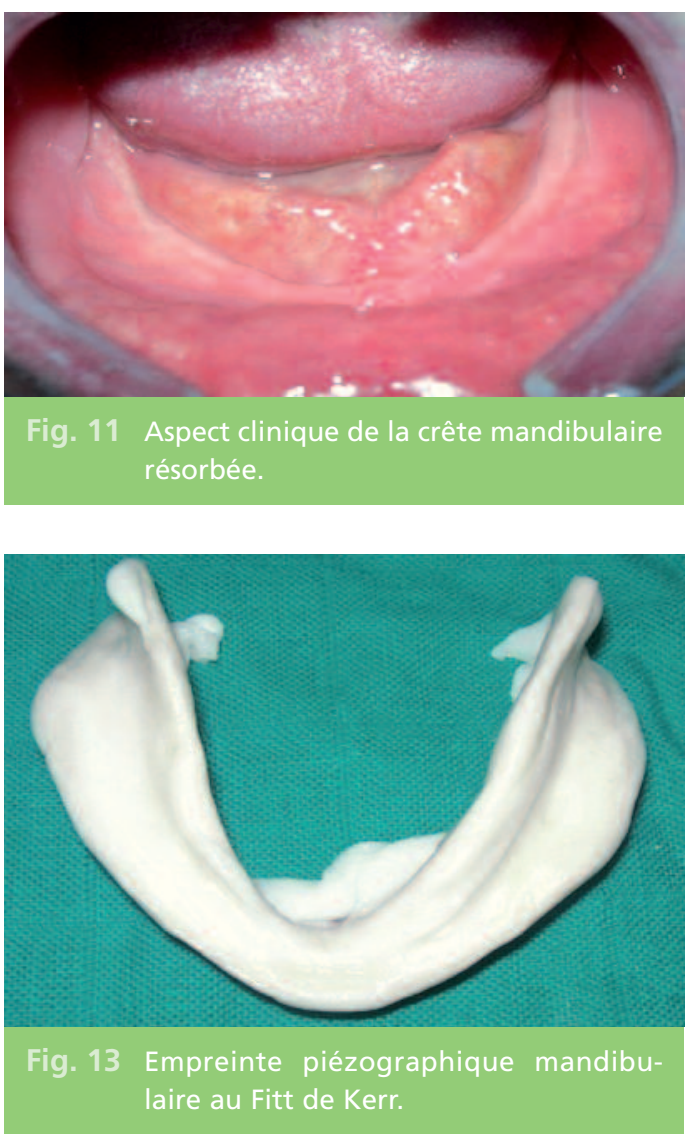
d'éviter un écrasement du matériau dans les sens vertical et horizontal).

Ce modelage sera rejeté après modelage définitif du côté opposé, puis refait car le patient a pu être surpris par le premier apport de matériau dans la cavité buccale, ce qui aurait faussé l'enregistrement avant de passer au modelage antérieur [14, 17].

Un moulage en négatif de la piézographie, formé d'une clé linguale et de deux clés vesti- bulaires, est réalisé en silicone. La face supérieure de ces clés doit être alignée sur le plan occlusal représenté par la surface supérieure de la piézographie.

Le montage des dents prothétiques est réalisé dans le volume délimité par les clés, réplique en négatif de la piézographie.

Une fois polymérisées, les prothèses sont essayées en bouche, témoignant une grande satisfaction du patient (fig. 14).

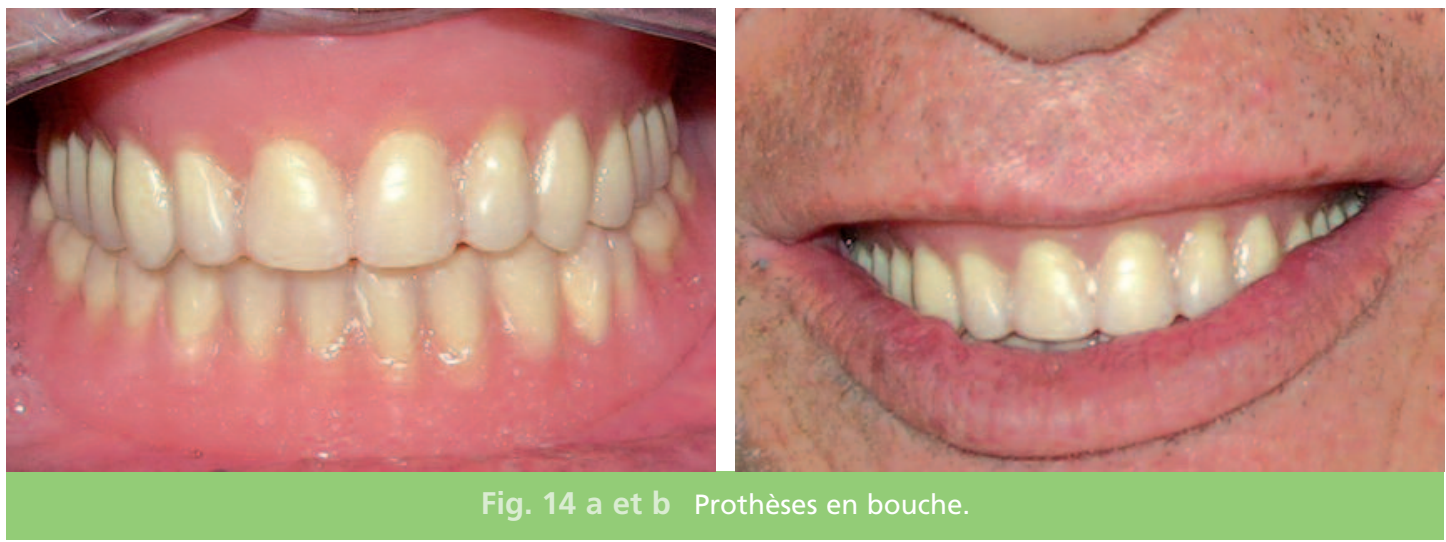

\section{Discussion}

Les techniques classiques d'empreintes apparaissent parfois insuffisantes, d'où l'idée d'exploiter l'action de la langue et des joues sur l'extrados prothétique, afin d'obtenir une stabilité de la prothèse, principale motivation de la piézographie.

En 1800, Gardette établissait le fait qu'une prothèse pouvait être stabilisée grâce aux contractions involontaires et instinctives des muscles de la cavité buccale.

Actuellement, les travaux de P. Klein en France, Heath en Angleterre, Schwindling en Allemagne, Beresin et Schiesser aux États-Unis convergent vers un même objectif : obtenir une sta- bilisation des prothèses mandibulaires grâce à I'utilisation de l'espace prothétique pour obtenir une parfaite symbiose en adaptant la forme à la fonction $[14,17]$.

La technique piézographique s'utilise principalement en prothèse amovible complète à l'arcade mandibulaire en raison de l'anatomie de crêtes fortement résorbées, voire sans aucun relief (classes III et IV d'Atwood) ; de I'ancienneté de la perte des organes dentaires, sans restauration prothétique, favorisant ainsi le développement des structures musculaires labiales, jugales et linguales et leur envahissement; de l'instabilité d'une prothèse amovible 
complète mandibulaire réalisée selon des méthodes traditionnelles; ou comme un complément de l'analyse pré-implantaire, toujours par le modelage effectué par les organes périprothétiques, pour évaluer l'importance des artifices de soutien tissulaire $[14,18]$.

Cependant, de nombreux auteurs ne sont pas favorables à ce type de prothèse en tant que prothèse définitive. Notamment J. Lejoyeux pour qui la piézographie n'est qu'une étape de la mise en condition tissulaire qu'il faut poursuivre progressivement par une extension de la surface de sustentation, et un élargissement de l'espace prothétique disponible jusqu'à pouvoir réaliser une prothèse classique [19].

La piézographie permet également la bonne réhabilitation prothétique des édentés complets présentant une paralysie faciale, ainsi que la potentialisation de la qualité des prothèses mandibulaires fixées sur des implants [20].

\section{Conclusion}

La piézographie, procédé déjà ancien de modelage appliqué en odontologie par Fish dans les années 1930, fait appel aux pressions produites par la langue et par la sangle musculaire buccino-labiale lors de la phonation pour délimiter le couloir prothétique où viendra se loger la prothèse.

Cette dernière s'intègre ainsi parfaitement au contexte physiologique de l'édenté total; elle occupe exactement l'espace prothétique disponible, ce qui assure son acceptation ainsi que sa pérennité contrairement à la méthode classique où la prothèse obtenue sera considérée comme un élément étranger par la musculature paraprothétique qui l'expulsera à chaque sollicitation.

De ce fait, le concept et la technique de la piézographie doivent être bien compris pour que le procédé devienne une nécessité maîtrisée au sein du cycle de la construction prothétique de l'édenté total, en particulier le gériatrique.

\section{Bibliographie}

1. Klein P.

Quelques définitions pour mieux comprendre la piézographie. Inf Dent 1989;71(29):2519-23.

2. Bernhardt $\mathrm{M}$ et al. Piézographie et plan d'occlusion en prothèse adjointe totale. Synergie Prothétique 2001;3(2):129-37.

3. Bernhardt $\mathrm{M}$ et al.
Quel peut être l'apport de la piézographie au montage fonctionnel en prothèse totale ? Stratégie Prothétique 2002;2(2):87-99.

4. Samoian R.

Apport des techniques piézographiques en prothèse adjointe totale. Act Odonto Stomatol 1992;177:157-77.
5. Klein $\mathrm{P}$ La piézographie. Rev Odonto Stomatol 1976;V:345-9.

6. Devin R. Empreintes phonétiques. Ann Odonto Stomatologiques 1961;6:231-50.

7. Budtz-Jorgensen E, Clavel R. Anatomie et physiologie faciale et buccale de l'édenté. La prothèse 
totale : théorie, pratique et aspects médicaux.

Paris : Masson 1995:24-9.

8. Taddei C, Wolfram-Gabel R, Waltmann E.

Prothèse amovible : anatomie de l'édenté total. Encycl Med Chir, (Paris-France) Stomat Odont II 1995;23-325-A-10.

9. Dubois J, Miquel $\mathrm{JL}$, Manciet G, Ferran P, Bousquet $A$.

La sénescence au niveau de la sphère orale. Chir Dent Fr 1983;53:36-9.

10. Revol P, Devoize L, Deschaumes C, Barthélémy I, Baudet-Pommel M, Mondié JM.

Stomatologie gériatrique. EMC (Elsevier SAS, Paris), Stomatologie 2006;22-052-A-10.

11. Jaudoin $P$, Millet $C$, Mifsud $S$. Empreintes en prothèse complète. Encycl Med Chir, Odontologie, 2006;23-325-C-10.
12. Klein $P$.

La piézographie

en prothèse adjointe

mandibulaire.

Paris :

Dactylo-Sorbonne édit., 1973.

13. Nabid A.

Espace prothétique mandibulaire

en prothèse adjointe totale

et piézographie.

Inf Dent 2000;30:2875-85.

14. Chevalier L.

Apport des techniques piézographiques dans l'exercice quotidien en prothèse amovible complète.

Thèse Doctorat d'État, Nancy : Univ Nancy I, 2007.

15. Miller WP, Monteith $B$, Heath MR.

The effect of variation of the lingual shape of mandibular complete dentures on lingual resistance to lifting forces. Gerodontology 1998;15(2):113-9.

16. Nabid A, Daoud FZ,
Benouniche M, Smadhi L. Le concept de " zone neutre d'équilibre " en prothèse totale mandibulaire. LQOS 1986;44:315-24.

17. Jardel V, Richard A, Hirigoyen M. Les empreintes piézographiques : évolution dans le choix des matériaux. Cah Proth 1992;79:27-35.

18. Nabid A, Klein P. Méthodologie du moulage de l'espace prothétique mandibulaire gérodontologique : technique phonétique. Odontologia 1984;6:31-44.

19. Lejoyeux J.

Empreintes tertiaires. Les empreintes dans le traitement de l'édentation totale. Paris : CDP, 1986:112-7.

20. Burli V, Teodreschu HN, Morarasu CS.

La fonction phonétique chez l'édenté total : analyse en fréquence. Cah Proth, 1994;88:63-8.

\section{SUMMARY}

\section{Corridor and prosthetic gerodontology : simple treatment for optimum stability}

\section{Anissa REGRAGUI, Amal SEFRIOUI, Faïza BENFDIL, Amar BENAMAR, Ahmed ABDEDINE \\ Keywords \\ - bone resorption - piezography - prosthetic corridor - senescence}

The process of aging is often accompanied in the mouth of a narrow prosthetic corridor related to the importance of bone resorption and the invasion of peripheral elements, the situation is thus defavorable for prosthetic realization.

The use of piezography in complete denture is to better meet the oral physiology in the elderly while providing maximum stability prosthetic.

The piezography takes up four challenges : to restore its natural and harmonious appearance to the face, to offer an occlusal plane, find a vertical dimension of occlusion, strengthen jaw relationships fitted for masticatory function.

A rational, ergonomic protocol is particularly suitable for individual difficult cases, allowing to define the prosthetic volume devoted to the location of the artificial teeth. This is what we will try to illustrate through two clinical cases. 


\section{Questions-Réponses}

1. Quelle est la fonction sollicitée la plus fiabe lors de l'empreinte piézographique (selon P. Klein) :

$\square$ a - la phonation.

$\square \mathrm{b}$ - la déglutition.

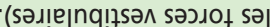

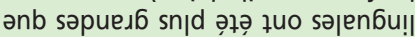

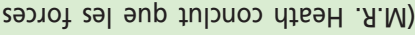

NON : uo!+!ฺn|бәр ер -

! (әәдddo|әләр sn|d ер әрело

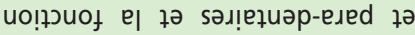

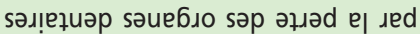

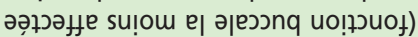

$$
\text { Ino : uo!feuoyd el - }
$$

\section{2. - La prise finale du matériau d'empreinte doit se faire :}

a - alors que la cavité buccale est au repos.

b - alors que la langue est en protraction. : əsuodẹy

\section{NON}

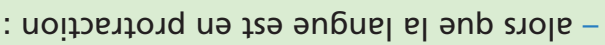
$\therefore$ (әц!ерпsnu әдq! -!nbə,

Ino

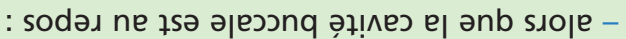
: әsuodə̧y

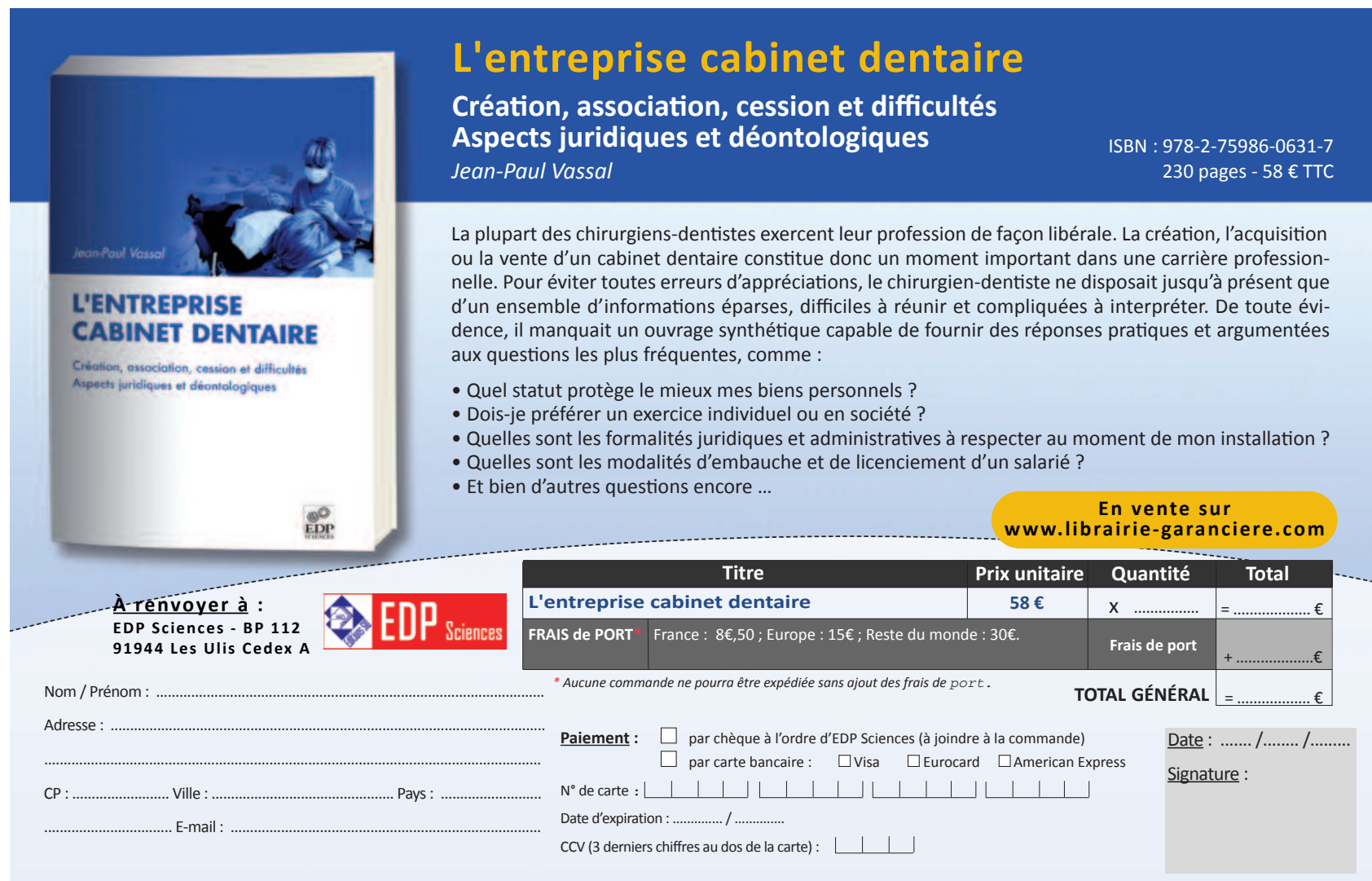

